\title{
The role of lung ultrasonography in predicting the clinical outcome of complicated community-acquired pneumonia in hospitalized children
}

\author{
Marcela Daniela Ionescu ${ }^{1,2}$, Mihaela Bălgrădean ${ }^{1,2}$, Cristina Filip ${ }^{2}$, Roxana Taraș ${ }^{1,2}$, \\ Georgiana Mihaela Căpitănescu², Florian Berghea ${ }^{1,3}$, Elena Camelia Berghea ${ }^{1,2}$, Cătălin \\ Gabriel Cîrstoveanu1,2
}

1"Carol Davila" University of Medicine and Pharmacy, ${ }^{2 "}$ Marie Curie" Emergency Children's Hospital,
3"Sfânta Maria" Clinical Hospital, Bucharest, Romania

\begin{abstract}
Aims: This study's objective was to analyze lung ultrasonography (LUS) characteristics in hospitalized pediatric patients with complicated community-acquired pneumonia (CAP). We hypothesized that LUS could be correlated with the clinical outcome in these cases. Materials and methods: In this retrospective study, we evaluated the LUS appearances (at admission and five days after the beginning of the treatment) and the progression of complicated CAP. Results: We identified 45 patients who fulfilled the inclusion criteria. Several complications occurred in these subjects during follow-up including: serofibrinous pleurisy $(62.2 \%)$, empyema $(15.6 \%)$, encapsulated pleurisy $(11.1 \%)$, lung abscess $(6.7 \%)$ and necrotizing pneumonia $(2.2 \%)$. In addition, $22.2 \%$ of the patients required surgical treatment: draining tube $(11.1 \%)$, decortication $(6.7 \%)$ and resection $(4.4 \%)$. Intensive care unit admission was needed in $8.9 \%$ of patients. The median duration of hospitalization was 14 [9.7; 19.7] days. The thickness of pleural effusion with a cut-off value of $10 \mathrm{~mm}$ seen by LUS was a predictor for the need for continuous thoracic drainage $(p<0.01)$, segmentectomy or thoracoscopic surgery $(p=0.03)$ and prolonged hospitalization over 10 days $(p<0.01)$. Hyperechogenic pleural effusion, presence of septa and fluid bronchogram on $1^{\text {st }}$ LUS evaluation were independent predictors of segmentectomy or thoracoscopic decortication $(p<0.01)$ and of longer hospitalization $(p=0.02, p<0.01, p<0.01$ respectively). Conclusions: The ultrasound characteristics of complicated CAP can offer valuable information to predict the clinical evolution of CAP and so can help the development of personalized medical management plans in these patients.
\end{abstract}

Keywords: lung; ultrasonography; paediatric; pneumonia; pleural effusion

\section{Introduction}

Community-acquired pneumonia (CAP) is a common infectious disease in children, involving the alveoli and variable proportion of pulmonary parenchyma, with po-

Received 21.02.2021 Accepted 22.06.2021

Med Ultrason

2022, Vol. 24, No 1, 19-26

Corresponding author: Elena Camelia Berghea

"Carol Davila" University of Medicine and

Pharmacy, "Marie Curie" Emergency

Children's Hospital, Bucharest, Romania

20 Bd Constantin Brâncoveanu,

75534 Bucharest, Romania

Phone: 0722565117

E-mail: bcamelia@gmail.com tentially severe and considerable morbidity and mortality. It is defined as the presence of signs and symptoms of pneumonia in a previously healthy child who acquired the infection outside of a healthcare setting [1,2]. The annual incidence of pneumonia in developed countries is estimated to be 40/1000 in children younger than 5 years of age and 20/1000 in children over 5 years of age [3]. Worldwide, CAP remains the largest cause of death in children and a major health issue. Approximately onehalf of children younger than 5 years of age with CAP require hospitalization. [1-3]. The true incidence of pneumonia is difficult to define because many other clinical entities that express low respiratory tract infections are labelled as "pneumonia". No single sign or symptom is pathognomonic for CAP in children and the diagno- 
sis may be challenging. Fever and cough in presenting pictures suggest pneumonia, but non-specific symptoms, such as abdominal pain and nuchal rigidity, may create significant difficulties [1]. The etiological diagnosis is difficult and neither clinical nor radiologic features distinguish between bacterial, atypical bacterial and viral pneumonia $[2,4]$. Pleural effusion, empyema, necrotizing pneumonia, lung abscess and pneumatocele may complicate CAP in children [5].

Although not recommended routinely for diagnosis in children, chest radiography (chest X-ray) is the most used investigation for detecting CAP lesions. The technique has certain limitations as non-severe pneumonia cases may show normal chest X-ray or just perihilar changes while minimal pleural effusion may go undetected $[1,3]$. Few studies have shown that radiological findings are associated with the severity of CAP in pediatric patients. Despite that, the prognostic role of chest radiography in children with CAP has not been established $[6,7]$.

Thereby, the clinicians' interest shifted towards lung ultrasonography (LUS), considered in time a safe and accessible option for diagnosing pneumonia and its complications [8]. Recent systematic reviews have verified the high accuracy of LUS for diagnostic of pneumonia, concluding that it is a useful imaging alternative to chest radiography inclusive in pediatric CAP $[9,10]$. Even if ultrasound's usefulness is limited in evaluating a wellaerated lung due to the imperfect transmission of the sound waves, it is an essential diagnostic tool in the presence of consolidations, allowing the evaluation of lung parenchyma [11]. LUS can detect small pulmonary lesions not visible on chest X-ray, suggesting that this is the preferred approach for assessing patients with small consolidations or small parapneumonic pleural effusion [6,9,12-15]. Fluid or aerial bronchogram may be well described, suggesting a consolidation process. LUS is also fairly useful in evaluating the pleural effusion, providing the best detail of the fluid nature, quantity, consistency, echogenicity. It can demonstrate the presence of internal septations, cellular debris, honeycombing, pleural thickening, or the lack of free movements with gravity, suggesting complications that cannot be well characterized on static X-ray or computer tomography images $[6,7,11]$. Besides, LUS can help guide any invasive diagnostic or therapeutic procedures [11].

This study aimed at evaluating the LUS characteristics of complicated CAP in hospitalized children at baseline and 5 days after beginning the antibiotic treatment and to assess the LUS value for predicting the clinical outcome in these children. Therefore, we examined which LUS findings were risk factors for a poor outcome in complicated CAP, requiring specific invasive thera- peutic procedures. The following outcomes were pursued: needing continuous thoracic drainage, requiring surgical interventions consisting of either segmentectomy or video-assisted thoracoscopic surgery decortication and demanding prolonged hospitalization over 10 days.

\section{Material and methods}

\section{Study design}

We conducted a retrospective, observational, unicentric study. Data were collected from electronic medical records of pediatric patients with the diagnosis of CAP requiring hospitalization between 2017-2019 in "Marie S. Curie" Emergency Children's Hospital, Bucharest, Romania. All patients' records and clinical information were analyzed anonymously. The study was conducted with the approval of the local Ethics Committee.

Patients' medical files were studied for demographic information, clinical and paraclinical data, treatment, clinical course, and discharge summary. The diagnosis of CAP was established according to the British Thoracic Society guidelines [1]. Clinical findings (fever, cough, chest pain, respiratory distress, gastrointestinal complaints and other symptoms, respiratory rate, oxygen saturation), laboratory tests (complete blood count, Creactive protein - CRP and procalcitonin level) and imagistic investigations (chest X-ray and LUS) at admission and during hospitalization were noted. All patients were hospitalized and a $5^{\text {th }}$ day LUS control was performed, while control blood investigations were carried out only when considered by the physician. The chest X-rays and LUS clips or images were digitally archived in the hospital informatic system and they were reanalyzed for this study by an experienced senior pediatric radiologist.

\section{Performance of LUS}

LUS was performed soon after complicated CAP was suspected, using the GE LogiqS8 ultrasound system, with convex (C1-6) and linear (L3-12) probes. Anterior, lateral and posterior intercostal spaces were examined in longitudinal and transverse sections with patients in supine and sitting positions.

All the enrolled patients underwent first LUS at admission and the second LUS 5 days after the beginning of treatment. Per center's capabilities, the second LUS was performed by the same person that made the first examination - this approach reduced possible interobserver variability. Findings were reported as normal or LUS features of pneumonia were recorded including: the presence of pulmonary parenchymal lesions (consolidation/ atelectasis), number, size and localization of lesions, the presence of bronchogram and its characteristics (air or fluid bronchogram), the presence and aspect of pleural 


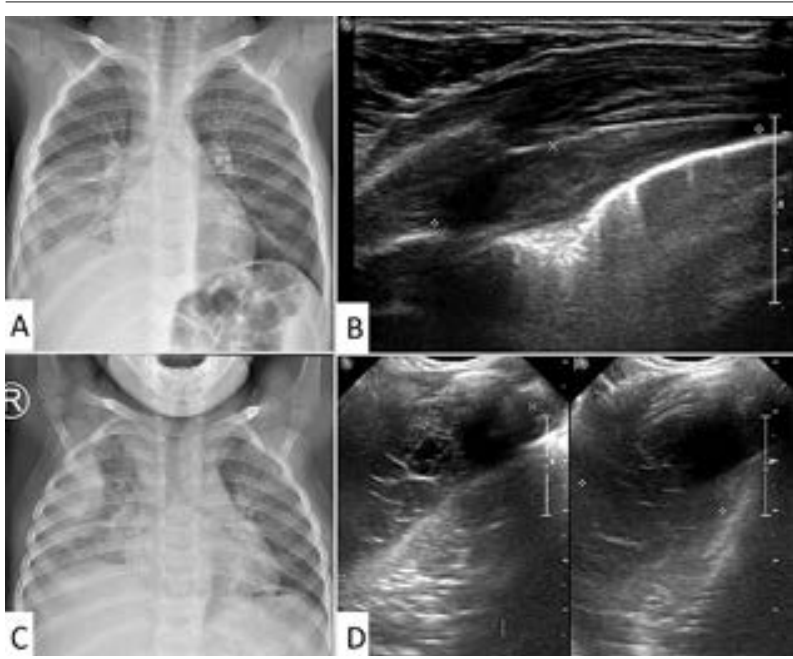

effusion (echogenicity, homogeneity, thickness, the presence of septa).

Two examples of comparative evolutive findings on the chest X-ray and LUS are presented in figure 1 and 2.

\section{Inclusion criteria}

We included pediatric patients (age under 18 years old) with admission diagnosis of complicated CAP, based on history, initial clinical examination and chest radiography, who underwent LUS at admission and 5 days after the beginning of antibiotic therapy.
Fig 1. A 2-year-old girl with severe community-acquired pneumonia who needed surgical treatment consisting of video-assisted thoracoscopic decortication, along with complex antibiotic and supportive therapy, requiring prolonged hospital stay over 10 days: A) Chest X-ray ( $\left.1^{\text {st }}\right)$ : right basal pulmonary opacity, with heterogeneous structure and air bronchogram, suggestive for right lower lobe consolidation; blunting the costal phrenic angle and right pleural homogenous opacity, with coastal intensity, net delimitation representative for pleural parapneumonic effusion; B) LUS (1 $\left.{ }^{\text {st }}\right)$ : right lateral-thoracic pleural effusion, with lenticular shape, echogenic appearance, and small free movements with gravity, having $3 \mathrm{~cm}$ length and a maximum thickness of $7 \mathrm{~mm}$; C) Chest X-ray (2nd): progression of lateral-thoracic opacity, with coastal intensity and tendency to lock on the lateral thoracic wall; D) LUS (2 ${ }^{\text {st }}$ ): right lateral-thoracic pleural effusion, with multiple cellular debris, septa and lack of free movements with gravity.

Severe CAP was considered according to the British Thoracic Society guidelines, including persistent fever, tachypnea, respiratory distress, cyanosis, grunting respirations, hypoxemia, tachycardia, prolonged capillary refill time, signs of dehydration or intense positively acute phase reactants (leukocytosis, CRP, procalcitonin) [1]. We defined as complicated CAP those patients presenting with clinical, biological and chest X-ray features suggestive for local development of pleural effusion or empyema, necrotizing pneumonia and lung abscess.

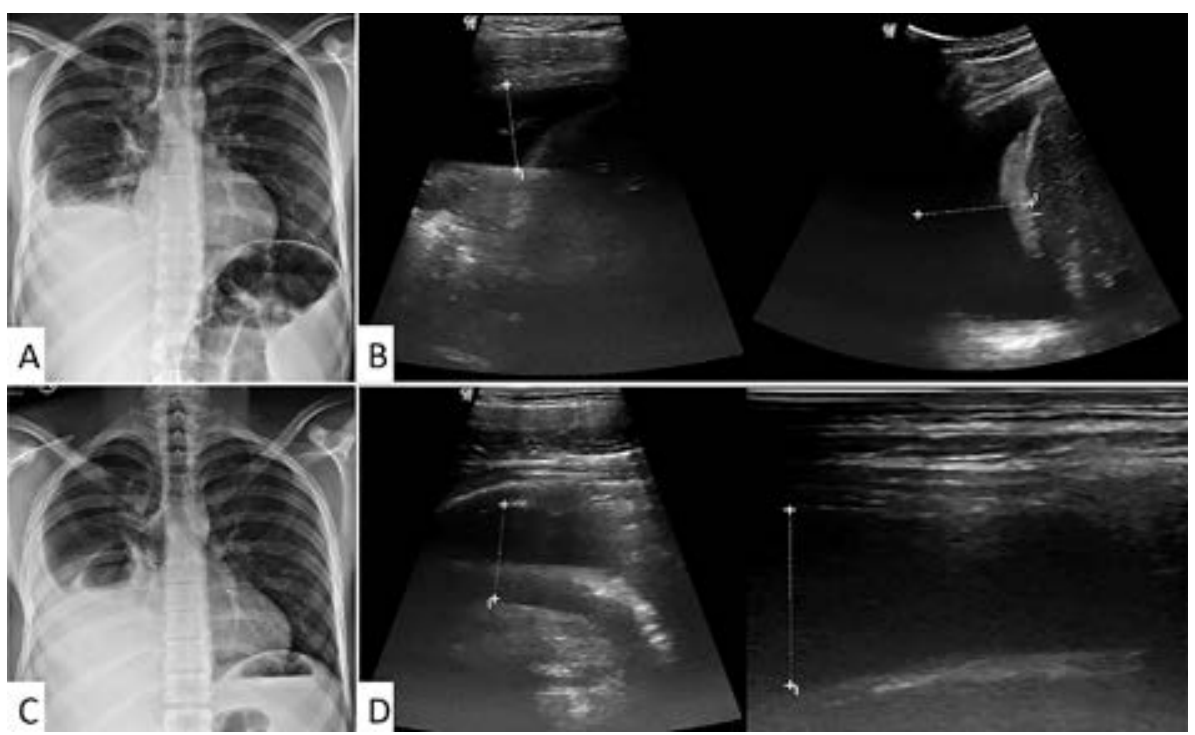

Fig 2. A 14-year-old boy with complicated community-acquired pneumonia who needed continuous thoracic drainage and complex antibiotic therapy: A) Chest X-ray ( $\left.{ }^{\text {st }}\right)$ : alveolar opacity with low intensity and flue contour of the inferior right pulmonary lobe; right basal pulmonary opacity, with homogenous structure, costal power, net delimitation and blunting of the costal phrenic angle, suggestive for medium pleural effusion, with minimal passive pulmonary collapse; B) LUS $\left(1^{\text {st }}\right)$ : right lateral-thoracic pleural effusion, with transonic liquid, in large quantity (up to axilla level in orthostatic position), determining passive lung collapse of the inferior right pulmonary lobe; C) Chest X-ray (2nd): slight progression of right pleurisy; D) LUS (2 ${ }^{\text {st }}$ ): persistent right lateral-thoracic pleural effusion, with transonic aspect, and free movements with gravity, without septa or cellular debris. 


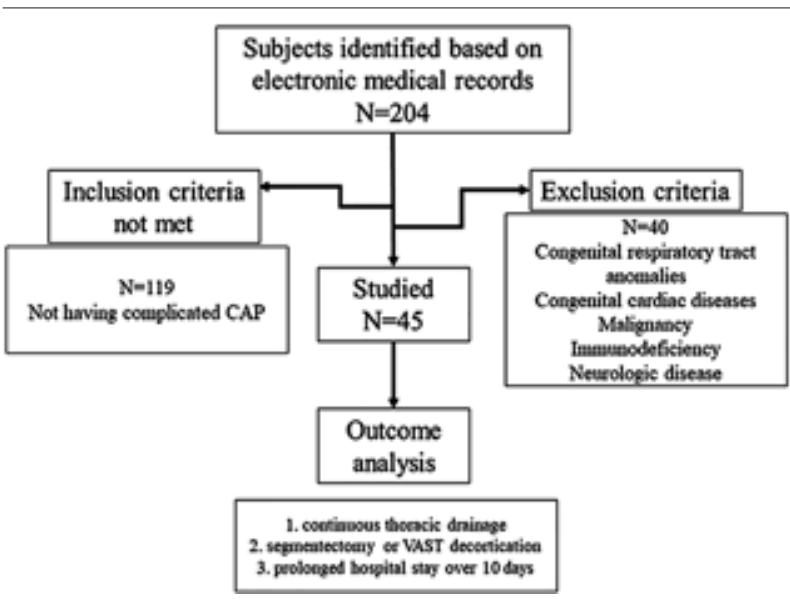

Fig 3. Flowchart of the study.

\section{Exclusion criteria}

Patients with underlying disease, including respiratory tract anomalies, malignancy, immunodeficiency, neurologic disorders (cerebral palsy, neuromuscular diseases), congenital heart disease, were excluded.

The flowchart of the study is presented in Fig 3.

\section{Statistical analysis}

We performed statistical analysis and graphs using the Analyze IT 5.5 program (Microsoft Office Excel Add-on, Leeds, UK). Continuous variables had a nongaussian distribution and were presented as the median and the interval between the quartiles. Categorical variables were presented as numbers and percentages. Differences in quantitative parameters were tested using nonparametric tests (Kruskal-Wallis). Qualitative data were compared with the chi-square test. We considered statistical significance at a p-value lower than 0.05.

\section{Results}

\section{Patients' characteristics}

Table I summarizes the main features of the included patients: demographic data, clinical and laboratory characteristics on admission, local and general complications and required major therapeutic procedures.

The evolution of the baseline pleural and pulmonary lesions (localization, size and aspect of lung consolidation and pleural effusion, presence and aspect of bronchogram) was appreciated using the second LUS assessment, performed after 5 days of treatment. Baseline radiologic and LUS findings 5 days after the beginning of treatment in all 45 patients are summarized in Table II.

\section{Relation between LUS characteristics and clinical outcome}

We analyzed the relation between LUS characteristics and clinical outcome. Using univariate analysis,
Table I. Cohort characteristics of the 45 patients enrolled.

\begin{tabular}{|c|c|}
\hline Parameter & Value \\
\hline Male gender & $30(66.7)$ \\
\hline Age (months) & $42[25.7 ; 69.7]$ \\
\hline Prior days of fever & $5[3 ; 7]$ \\
\hline Prior days of cough & $5[4 ; 7.3]$ \\
\hline Grunting & $9(20)$ \\
\hline Chest pain & $8(17.8)$ \\
\hline Respiratory rate (breaths/minute) & $40[30 ; 46.7]$ \\
\hline \multicolumn{2}{|l|}{ Respiratory distress } \\
\hline Minimum & $12(26.7)$ \\
\hline Moderate & $23(51.1)$ \\
\hline Severe & $10(22.2)$ \\
\hline Oxygen saturation & $94[90.6 ; 97]$ \\
\hline \multicolumn{2}{|l|}{ Other associated symptoms } \\
\hline Sleepiness & $5(11.1)$ \\
\hline Loss of appetite & $10(22.2)$ \\
\hline Diarrhea & $1(2.2)$ \\
\hline Vomiting & $8(17.8)$ \\
\hline Abdominal pain & $3(6.7)$ \\
\hline None & $18(40)$ \\
\hline White blood cell count $\left(\mathrm{x} 10^{3}\right)$ & $19000[14566 ; 24083]$ \\
\hline PMN & $78[64.7 ; 85]$ \\
\hline CRP (mg/dL) & $145[76.3 ; 282]$ \\
\hline \multicolumn{2}{|l|}{ Procalcitonin (ng/dL) } \\
\hline$<0.5$ & $17(37.8)$ \\
\hline $0.5-2$ & $6(13.3)$ \\
\hline $2-10$ & $10(22.2)$ \\
\hline$>10$ & $12(26.7)$ \\
\hline Severe CAP & $18(40)$ \\
\hline \multicolumn{2}{|l|}{ Local complications } \\
\hline Serofibrinous pleurisy & $28(62.2)$ \\
\hline Empyema & $7(15.6)$ \\
\hline Encapsulated pleurisy & $5(11.1)$ \\
\hline Lung abscess & $3(6.7)$ \\
\hline Necrotizing pneumonia & $1(2.2)$ \\
\hline \multicolumn{2}{|l|}{ General complications } \\
\hline SIRS & $6(13.3)$ \\
\hline Sepsis & $23(51.1)$ \\
\hline Severe sepsis & $2(4.4)$ \\
\hline Septic shock & $1(2.2)$ \\
\hline Surgery & $10(22.2)$ \\
\hline Draining tube & $5(11.1)$ \\
\hline Thoracotomy and decortication & $3(6.7)$ \\
\hline Surgical resection & $2(4.4)$ \\
\hline $\mathrm{ICU}$ & $4(8.9)$ \\
\hline Hospital stay (days) & $14[9.7 ; 19.7]$ \\
\hline Sequelae $<6$ months & $15(33.3)$ \\
\hline Pahipleuritis & $13(28.9)$ \\
\hline Segmentectomy & $2(4.4)$ \\
\hline
\end{tabular}

Continuous variables are presented as median [IQR]. Categorical variables are presented as number $(\%) . \mathrm{CRP}=\mathrm{C}$ reactive protein; $\mathrm{PMN}=$ polymorphonuclear leucocytes; ICU = Intensive Care Unit; $\mathrm{IQR}=$ Interquartile Range; SIRS = Systemic Inflammatory Response Syndrome 
we examined which LUS findings were risk factors for a poor outcome in complicated CAP. We considered the poor outcome at least one of the three following condi-

Table II. Cohort imagistic features of the 45 patients enrolled

\begin{tabular}{ll}
\hline Parameter & Value \\
\hline Chest radiograph (at admission) & \\
Multilobed consolidation & $3(6.7)$ \\
Pleural effusion & $40(88.9)$ \\
Lung abscess & $2(4.4)$ \\
Consolidation & $42(93.3)$ \\
$\mathbf{1}^{\text {st }}$ LUS (at admission) & \\
Consolidation & $43(95.6)$ \\
Pleural effusion & $45(100)$ \\
$\quad$ Thickness (mm) & $7[5 ; 15]$ \\
$\quad$ Echogenicity & \\
$\quad$ Isoechogenic fluid & $15(33.3)$ \\
$\quad$ Hyperechogenic fluid $\quad$ & $8(17.8)$ \\
$\quad$ Transonic fluid & $22(48.9)$ \\
$\quad$ Septa & $32(71.1)$ \\
Multilobed lesions & $13(28.9)$ \\
Air bronchogram & $8(17.8)$ \\
Fluid bronchogram & $26(57.8)$ \\
$\mathbf{2}^{\text {nd }}$ LUS (5 days after admission) & $16(35.6)$ \\
Consolidation & \\
Improvement $v s .1^{\text {st }}$ & $23(56.1)$ \\
Pleural effusion & $21(46.7)$ \\
Improvement $v s .1^{\text {st }}$ & $17(41.5)$ \\
\hline Continuous variables are presented as median & {$[\mathrm{IQR}]$. Categorical } \\
variables are presented as number (\%) &
\end{tabular}

tions: the need for continuous thoracic drainage, indication for surgical interventions (consisting of either segmentectomy or video-assisted thoracoscopic surgery decortication), and requiring prolonged hospitalization over 10 days.

Regarding the first outcome, we noted that LUS could predict the need for continuous thoracic drainage in complicated CAP at a pleural effusion thickness cut-off value of $10 \mathrm{~mm}$. The ultrasound identification of aerial bronchogram had a statistically significant protective value regarding the need for continuous thoracic drainage. Those patients often presented a good clinical outcome and did not need invasive therapeutic procedures, including thoracic drainage. The fluid bronchogram was dominant in patients that required continuous thoracic drainage, alongside conservative treatment (Table III).

Concerning the second outcome, the need for surgical interventions including segmentectomy or videoassisted thoracoscopic surgery decortication, we found that the hyperechogenic pleural effusion, the thickness of pleural effusion with a cut-off value of $10 \mathrm{~mm}$, the presence of septa and the fluid bronchogram on $1^{\text {st }}$ LUS evaluation predicted a poor outcome and a worse clinical evolution, requiring surgical therapeutic procedures. On the other hand, the presence of aerial bronchogram and the homogenous pleural effusion alongside the improved appearance of pleural effusion on the $2^{\text {nd }}$ LUS evaluation were associated with a better clinical course (Table IV).

Using univariate analyses, it was also examined whether LUS findings were risk factors for a poor out-

Table III. Association between LUS findings and the need for continuous thoracic drainage

\begin{tabular}{|c|c|c|c|c|c|}
\hline \multirow[t]{2}{*}{ Parameter } & \multicolumn{2}{|c|}{ Thoracic tube drainage } & \multicolumn{2}{|c|}{ Univariate analysis } & \multirow[t]{2}{*}{ p-value } \\
\hline & Yes (5) & No (40) & Odds ratio & $95 \%$ confidence interval & \\
\hline \multicolumn{6}{|l|}{$1^{\text {st }}$ LUS (at admission) } \\
\hline Consolidation & $4(80)$ & $39(97.5)$ & 0.1 & $0.009-1.19$ & 0.07 \\
\hline Hyperechogenic fluid & $2(40)$ & $6(15)$ & 3.77 & $0.61-24.52$ & 0.16 \\
\hline Homogeneity & $2(40)$ & $30(75)$ & 0.22 & $0.03-1.32$ & 0.1 \\
\hline Thickness $>10 \mathrm{~mm}$ & $5(100)$ & $12(30)$ & 2.66 & $2.66-$ & $<0.01$ \\
\hline Septa & $3(60)$ & $10(25)$ & 4.5 & $0.75-26.43$ & 0.1 \\
\hline Multifocal lesions & $2(40)$ & $6(85)$ & 0.26 & $0.04-1.63$ & 0.16 \\
\hline Aerial Bronchogram & $0(0)$ & $26(65)$ & 2.13 & $2.13-$ & $<0.01$ \\
\hline Fluid Bronchogram & $4(80)$ & $12(30)$ & 0.1 & $0.01-0.83$ & 0.02 \\
\hline \multicolumn{6}{|c|}{$2^{\text {nd }}$ LUS (5 days after admission) } \\
\hline Consolidation & $4(80)$ & $19(52.5)$ & 4.42 & $0.57-32.06$ & 0.17 \\
\hline Improvement vs. $1^{s t}$ & $1(20)$ & $20(50)$ & 4 & $0.52-29.01$ & 0.2 \\
\hline Pleural effusion & $5(100)$ & $12(30)$ & 2.66 & $2.66-$ & $<0.01$ \\
\hline Improvement vs. $1^{s t}$ & $0(0)$ & $25(62.5)$ & 1.92 & $1.92-$ & $<0.01$ \\
\hline Severe CAP & $4(80)$ & $14(35)$ & 7.42 & $0.96-54.25$ & 0.052 \\
\hline
\end{tabular}

Data are expressed as numbers $(\%)$. CAP $=$ community acquired pneumonia; LUS = lung ultrasonography 
come, including prolonged hospitalization over 10 days. For this outcome, we noted a poor evolution regarding hyperechogenic pleural effusion, the thickness of pleural effusion with a cut-off value of $10 \mathrm{~mm}$, the presence of septa and the fluid bronchogram on $1^{\text {st }}$ LUS evaluation (Table V). The presence of aerial bronchogram and the homogenous pleural effusion alongside the improved appearance of pleural effusion on the $2^{\text {nd }}$ LUS evaluation predicted a better course for this outcome.

\section{Discussion}

Our study analyzes the LUS characteristics of complicated CAP in hospitalized pediatric patients at admission (baseline) and after the beginning of antibiotic treatment. In addition, the study highlights how specific LUS features may predict the clinical course of these patients by assessing the following three outcomes: the need for continuous thoracic drainage, requiring surgi-

Table IV. Association between LUS findings and the need for surgery

\begin{tabular}{|c|c|c|c|c|c|}
\hline \multirow[t]{2}{*}{ Parameter } & \multicolumn{2}{|l|}{ Surgery } & \multicolumn{2}{|c|}{ Univariate analysis } & \multirow[t]{2}{*}{ p-value } \\
\hline & Yes (5) & No (40) & Odds ratio & $95 \%$ confidence interval & \\
\hline \multicolumn{6}{|l|}{$1^{\text {st }}$ LUS } \\
\hline Consolidation & $5(100)$ & $38(95)$ & NS & & 0.6 \\
\hline Hyperechogenic fluid & $3(60)$ & $5(12.5)$ & 10.5 & $1.6-68.8$ & $<0.01$ \\
\hline Homogeneity & $1(20)$ & $31(77.5)$ & 0.07 & $0.01-0.58$ & $<0.01$ \\
\hline Thickness $>10 \mathrm{~mm}$ & $4(80)$ & $13(32.5)$ & 8.3 & $1.07-60.91$ & 0.03 \\
\hline Septa & $4(80)$ & $9(22.5)$ & 13.77 & $1.72-103.17$ & $<0.01$ \\
\hline Multifocal lesions & $0(0)$ & $8(80)$ & NS & & 0.27 \\
\hline Aerial bronchogram & $0(0)$ & $26(65)$ & 2.13 & $2.13-$ & $<0.01$ \\
\hline Fluid bronchogram & $5(100)$ & $11(27.5)$ & 0 & $0-0.33$ & $<0.01$ \\
\hline \multicolumn{6}{|l|}{$2^{\text {nd }}$ LUS } \\
\hline Consolidation & $4(80)$ & $19(47.5)$ & 4.42 & $0.57-32.06$ & 0.17 \\
\hline Improvement vs. $1^{\text {st }}$ & $1(20)$ & $20(50)$ & 4 & $0.52-29.01$ & 0.2 \\
\hline Pleural effusion & $5(100)$ & $12(30)$ & 2.66 & $2.66-$ & $<0.01$ \\
\hline Improvement vs. $1^{\text {st }}$ & $0(0)$ & $25(62.5)$ & 1.92 & $1.92-$ & $<0.01$ \\
\hline Severe CAP & $4(80)$ & $14(35)$ & 7.42 & $0.96-54.25$ & 0.052 \\
\hline
\end{tabular}

Data are expressed as numbers (\%). NS: not significant; CAP = community acquired pneumonia; LUS = lung ultrasonography

Table V. Association between LUS findings and more extended hospital stay

\begin{tabular}{|c|c|c|c|c|c|}
\hline \multirow[t]{2}{*}{ Parameter } & \multicolumn{2}{|c|}{ Hospital stays over 10 days } & \multicolumn{2}{|c|}{ Univariate analysis } & \multirow[t]{2}{*}{ p-value } \\
\hline & Yes (30) & No (15) & Odds ratio & $95 \%$ confidence interval & \\
\hline \multicolumn{6}{|l|}{$1^{\text {st }}$ LUS, number, (\%) } \\
\hline Consolidation & $29(96.2)$ & $14(93.3)$ & 2.07 & $0.19-21.72$ & 0.6 \\
\hline Hyperechogenic fluid & $8(26.7)$ & $0(0)$ & 1.27 & $1.27-$ & 0.02 \\
\hline Homogeneity & $17(56.7)$ & $0(0)$ & 0 & $0-0.37$ & $<0.01$ \\
\hline Thickness $>10 \mathrm{~mm}$ & $16(53.3)$ & $1(6.7)$ & 16 & $2.26-106.27$ & $<0.01$ \\
\hline Septa & $13(43.3)$ & $0(0)$ & 2.68 & $2.68-$ & $<0.01$ \\
\hline Multifocal lesions & $6(20)$ & $2(13.3)$ & 0.61 & $0.12-3.2$ & 0.58 \\
\hline Aerial bronchogram & $14(46.7)$ & $12(80)$ & 4.57 & $1.1-18.38$ & 0.038 \\
\hline Fluid bronchogram & $16(53.3)$ & $0(0)$ & 0 & $0-0.25$ & $<0.01$ \\
\hline \multicolumn{6}{|l|}{$2^{\text {nd }}$ LUS, number, (\%) } \\
\hline Consolidation & $18(60)$ & $5(33.3)$ & 3 & $0.81-10.74$ & 0.091 \\
\hline Improvement vs. $1^{\text {st }}$ & $12(40)$ & $9(60)$ & 2.25 & $0.64-7.85$ & 0.2 \\
\hline Pleural effusion & $14(46.7)$ & $3(20)$ & 3.5 & $0.84-14.04$ & 0.08 \\
\hline Improvement vs. $1^{\text {st }}$ & $13(43.3)$ & $12(80)$ & 5.23 & $1.26-21.09$ & 0.019 \\
\hline Severe CAP & $18(60)$ & $0(0)$ & 5.19 & 5.19- & $<0.01$ \\
\hline
\end{tabular}

Data are expressed as numbers $(\%) . \mathrm{CAP}=$ community acquired pneumonia; LUS = lung ultrasonography 
cal treatment, and the need for prolonged hospitalization over 10 days. To our knowledge, this is the first study that evaluated the predictive potential of the LUS characteristics in hospitalized pediatric patients with complicated CAP. Multiple studies have mainly focused on LUS accuracy compared with chest X-ray findings in diagnosing and evaluating pediatric CAP, offering very promising results (overall high sensitivity - up to 95\% - and high specificity - up to $96 \%$ ) $[1,2,9,16-18]$. Also, few studies compared LUS with chest CT, claiming the accuracy of LUS in the evaluation of CAP and its complications inclusively in pediatric patients $[19,20]$.

All patients enrolled in our study presented local complications of CAP, including serofibrinous pleurisy, encapsulated pleurisy, pulmonary abscess or necrotizing pneumonia. LUS evidence of pulmonary consolidation with fluid bronchogram, hyperechogenic pleural fluid and septa was demonstrated to be statistically significant predictors of poor clinical outcome, expressed as prolonged hospitalization (more than 10 days), the need for continuous thoracic drainage and the need for segmentectomy or video-assisted thoracoscopy surgery decortication.

The fluid bronchogram, generally described in approximately $20 \%$ of CAP patients, less frequently than the air bronchogram, represents an exudate packed conducting airway that occurs in the early phase of the disease because of bronchial obstruction with secretions and edema $[14,21,22]$. Our study described the presence of fluid bronchogram as a negative predictor of complicated CAP, needing surgical treatment and prolonged hospitalization $(p<0.01)$. Therefore, this has allowed for the conclusion that a fluid bronchogram revealed on LUS should indicate the need to consider CAP's potential progression to complications requiring supplemental therapeutic measures.

However, the most characteristic sign of CAP remains the festive air bronchogram which is generally detectable in about $70-97 \%$ of cases, reflecting the residual air within the consolidation areas $[6,23,24]$. While the fluid bronchogram dominated the positive outcome group in our study, the presence of air bronchogram on LUS performed on admission had a statistically significant protective value $(\mathrm{p}<0.05)$.

Because a liquid allows for excellent propagation of sound waves, LUS is a particularly good modality to assess the presence of parapneumonic pleural effusions. The role of LUS in assessing pleural effusion is very well known, as well as the prediction of poor outcomes when complex pleural lesions are detected, based mainly on adult studies $[9,18]$. Our paper confirms these findings and, in addition, it highlights the importance of LUS in assessing CAP-associated pleural effusion in pediat- ric patients. Also, the study evaluates ultrasound pleural effusion features in the early phase of CAP (admission time) and after initiation of antibiotic therapy. We identified hyperechogenic pleural effusion and presence of septa on $1^{\text {st }}$ LUS evaluation as independent predictors of segmentectomy or thoracoscopic decortication $(p<0.01)$ and of prolonged hospitalization, over 10 days $(p=0.02$, $\mathrm{p}<0.01$, respectively). The thickness of pleural effusion with a cut-off value of $10 \mathrm{~mm}$ seen by LUS was also associated with requiring continuous thoracic drainage $(\mathrm{p}<0.01)$, segmentectomy or thoracoscopic surgery $(\mathrm{p}=0.03)$ and prolonged hospitalization over 10 days $(p<0.01)$. In the second LUS evaluation, we found that the alleviated pleurisy predicted a better outcome with a statistically significant relationship between the LUS characteristics and clinical outcome.

Based on these findings, our study supports the consideration of LUS for diagnosis and monitoring of complicated CAP in hospitalized children and also suggests that LUS may be a good early predictor of poor clinical outcome in these patients Taken all together, our results may impact daily clinical practice, by providing new data to monitor treatment response, alongside with current follow-up clinical (fever remission) and biological (leukocytosis, CRP and procalcitonin level reduction) parameters.

\section{Study limits and strengths}

The study included a relatively small number of children, although it represents the entire patient population (satisfying inclusion and exclusion criteria) from one of the largest children's hospitals. The unicentric design offered the chance of a better-standardized work; simultaneously, the execution of LUS by a single sonographer might raise the question of generalizability in the case of less experienced hands. The retrospective design reduced the control of the quality of available data. Although, our study strengthens the role of LUS for early detection, monitoring and reliable prediction of severe outcome in complicated pediatric CAP. LUS has proven to be a useful tool to avoid more invasive interventions and complex investigations, which could involve consuming more significant material and human resources.

\section{Conclusions}

In conclusion, our study provides a detailed LUS analysis as an advantageous imaging predicting and monitoring tool in pediatric patients hospitalized for complicated CAP. It highlights early LUS features that may predict poor clinical outcome in these patients. The results can help physicians better manage a child with CAP and offer a personalized approach, from diagnosis to treatment and 
follow-up. However, more extensive prospective studies are necessary to support our findings regarding the association between LUS and clinical outcome in pediatric patients with complicated CAP.

\section{Conflict of interests: none}

\section{References}

1. Harris M, Clark J, Coote N, et al. British Thoracic Society guidelines for the management of community-acquired pneumonia in children: update 2011. Thorax 2011;66:ii1ii23.

2. Man SC, Fufezan O, Sas V, Schnell C. Performance of lung ultrasonography for the diagnosis of community-acquired pneumonia in hospitalised children. Med Ultrason 2017;19:276-281.

3. Everard ML. Paediatric respiratory infections. Eur Respir Rev 2016;25:36-40.

4. Heiskanen-Kosma T, Korppi M, Jokinen C, et al. Etiology of childhood pneumonia: serologic results of a prospective, population-based study. Pediatr Infect Dis J 1998;17:986991.

5. Mbata G, Chukwuka C, Onyedum C, Onwubere B, Aguwa E. The Role of Complications of Community-Acquired Pneumonia on the Outcome of the Illness: A Prospective Observational Study in a Tertiary Institution in Eastern Nigeria. Ann Med Health Sci Res 2013;3:365-369.

6. Reissig A, Gramegna A, Aliberti S. The role of lung ultrasound in the diagnosis and follow-up of communityacquired pneumonia. Eur J Intern Med 2012;23:391-397.

7. Chen IC, Hsu JH, Wu JR, Dai ZK. Updated Guidelines for Childhood Pneumonia Management: A Promising Role for Lung Ultrasound. Pediatr Neonatol 2015;56:363-364.

8. Weinberg B, Diakoumakis EE, Kass EG, Seife B, Zvi ZB. The air bronchogram: sonographic demonstration. AJR Am J Roentgenol 1986;147:593-595.

9. Heuvelings CC, Bélard S, Familusi MA, Spijker R, Grobusch MP, Zar HJ. Chest ultrasound for the diagnosis of paediatric pulmonary diseases: a systematic review and meta-analysis of diagnostic test accuracy. Br Med Bull 2019;129:35-51.

10. Xin H, Li J, Hu HY. Is Lung Ultrasound Useful for Diagnosing Pneumonia in Children? A Meta-Analysis and Systematic Review. Ultrasound Q 2018;34:3-10.

11. Light M, Blaisdell CJ, Homnick DN, Schechter MS, Weinberger MW. (Eds.). Paediatric Pulmonology. American Academy of Pediatrics, 2011.
12. Claes AS, Clapuyt P, Menten R, Michaux N, Dumitriu D. Performance of chest ultrasound in paediatric pneumonia. Eur J Radiol 2017;88:82-87.

13. Esposito S, Papa SS, Barzani I, et al. Performance of lung ultrasonography in children with community-acquired pneumonia. Ital J Pediatr 2014;40:37.

14. Shah VP, Tunik MG, Tsung JW. Prospective Evaluation of Point-of-Care Ultrasonography for the Diagnosis of Pneumonia in Children and Young Adults. JAMA Pediatr 2013;167:119-125.

15. Reali F, Sferrazza Papa GF, Carlucci P, et al. Can lung ultrasound replace chest radiography for the diagnosis of pneumonia in hospitalised children? Respiration 2014;88:112115.

16. Musolino AM, Tomà P, Supino MC, et al. Lung ultrasound features of children with complicated and noncomplicated community-acquired pneumonia: A prospective study. Pediatr Pulmonol 2019;54:1479-1486.

17. Pereda MA, Chavez MA, Hooper-Miele CC, et al. Lung Ultrasound for the Diagnosis of Pneumonia in Children: A Meta-analysis. Paediatrics 2015;135:714-722.

18. Ambroggio L, Sucharew H, Rattan MS, et al. Lung Ultrasonography: A Viable Alternative to Chest Radiography in Children with Suspected Pneumonia? J Pediatr 2016;176:93-98.e7.

19. Saraya S, El Bakry R. Ultrasound: Can it replace CT in the evaluation of pneumonia in paediatric age group? Egypt J Radiol Nucl Med 2017;48:687-694.

20. Kurian J, Levin TL, Han BK, Taragin BH, Weinstein S. Comparison of Ultrasound and CT in the Evaluation of Pneumonia Complicated by Parapneumonic Effusion in Children. AJR Am J Roentgenol 2009;193:16481654.

21. Chen IC, Lin MY, Liu YC, et al. The role of transthoracic ultrasonography in predicting the outcome of communityacquired pneumonia in hospitalised children. PLoS One 2017;12:e0173343.

22. Haggag YI, Mashhour K, Ahmed K, Samir N, Radwan W. Effectiveness of Lung Ultrasound in Comparison with Chest X-Ray in Diagnosis of Lung Consolidation. Open Access Maced J Med Sci 2019;7:2457-2461.

23. Sperandeo M, Carnevale V, Muscarella S, et al. Clinical application of transthoracic ultrasonography in patients with pneumonia. Eur J Clin Invest 2011;41:1-7.

24. Byington CL, Spencer LY, Johnson TA, et al. An epidemiological investigation of a sustained high rate of paediatric parapneumonic empyema: risk factors and microbiological associations. Clin Infect Dis 2002;34:434-440. 